\title{
Photopolymerization of Methyl Methacrylate with $N$-Benzylpyridinium Thiocyanate
}

\author{
Kenzo TABUCHI and Naokazu SAKotA* \\ Department of Industrial Chemistry, Niihama Technical College, \\ 7-1 Yagumo-cho, Niihama 792, Japan \\ *Sugai Chemical Co., Ltd., 4-4-6 Uzu, Wakayama 641, Japan
}

(Received April 7, 1983)

\begin{abstract}
The photopolymerization of methyl methacrylate (MMA) with $N$-benzylpyridinium thiocyanate (BPT) was studied. The photopolymerization of MMA with BPT in dichloromethane gave an oligo(methyl methacrylate) (OMMA), which was presumably formed by initiation with thiocyano radical and termination with $\mathrm{N}$-benzylpyridinyl radical. The photopolymerization was also accelerated by the addition of carbon tetrachloride, which captured effectively the pyridinyl radical as was also the case with $N$-benzylpyridinium bromide. Photopolymerization of MMA with $\mathrm{N}$-benzylpyridinium chloride (BPC)- $\mathrm{KSCN}-\mathrm{CCl}_{4}$ in an aqueousorganic two-phase was also examined. The results suggested the following BPC-recycling photoinitiation mechanism. BPT formed by the ion exchange reaction between BPC and KSCN in an aqueous layer photodecomposed to the $\cdot \mathrm{SCN}$ and $N$-benzylpyridinyl radical. The latter reacted with carbon tetrachloride to produce $\mathrm{BPC}$ and $\cdot \mathrm{CCl}_{3}$. Both $\cdot \mathrm{SCN}$ and $\cdot \mathrm{CCl}_{3}$ initiated the polymerization of MMA, and $\mathrm{BPC}$ was transferred to the aqueous layer. Photopolymerization in aqueous-organic two-phase was also remarkably accelerated in an emulsified system with sodium dodecyl sulfate or $N$-cetylpyridinium bromide, each of which increased the interface area between the aqueous and organic layers and facilitated the phase-transfer.
\end{abstract}

KEY WORDS Photopolymerization / Methyl Methacrylate / N-

Benzylpyridinium Thiocyanate / $N$-Benzylpyridinium Chloride / Carbon

Tetrachloride / Patassium Thiocyanate / Sodium Dodecyl Sulfate / NCetylpyridinium Bromide /

The authors reported that the photopolymerization of methyl methacrylate (MMA) was remarkably sensitized by the charge transfer (CT) complex of $N$-benzylpyridinium bromide (BPB) in dichloromethane to give an oligo (methyl methacrylate) (OMMA), and that the polymerization was initiated with bromine atom and terminated with $N$ benzylpyridinyl radical. ${ }^{1}$ The photopolymerization of MMA with BPB was accelerated by the addition of carbon tetrachloride, which captured effectively $\mathrm{N}$-benzylpyridinyl radical to produce $\mathrm{N}$ benzylpyridinium chloride (BPC) and trichloromethyl radical to give a poly(methyl methacrylate) (PMMA) having a relatively large molecular weight. $^{2}$ The photopolymerization of MMA with $\mathrm{BPB}-\mathrm{CCl}_{4}$ system was also confirmed to be transformed into the one with the $\mathrm{BPC}-\mathrm{CCl}_{4}$ system soon after irradiation. ${ }^{2} N$-Benzylpyridinium thiocyanate (BPT) as well as BPB and BPC, is also expected to serve as a good photoinitiator in the polymerization of MMA, since the thiocyanate ion $^{3}$ has a greater electron-donating capacity than chloride and bromide ions, and the thiocyano radical ${ }^{4}$ can effectively initiate the polymerization of MMA.

BPB and BPC are easily soluble in water but only slightly in most organic solvents except methanol. But BPT is appreciably soluble in organic solvents and can thus be obtained by extraction with an appropriate organic solvent from an aqueous solution (of BPC) containing excess potassium thiocyanate. Accordingly, the photopolymerization of MMA with BPC- $\mathrm{KSCN}-\mathrm{CCl}_{4}$ in an aqueous system should proceed through a phase-transfer photoinitiation in aqueous-organic phases. 
In this paper, the authors discuss the photopolymerization of MMA with BPT in dichloromethane and also that in aqueous-organic twophase and emulsified systems.

\section{EXPERIMENTAL}

\section{Materials}

MMA was purified by the usual method immediately before use. BPB and BPC were prepared by the Menschutkin reaction. Carbon tetrachloride and dichloromethane were purified by the usual methods. Water was deionized and distilled. A special reagent grade of potassium thiocyanate was used. Sodium dodecyl sulfate (SDS) and $N$ cetylpyridinium bromide (CPB) were purchased from Wako Pure Chemical Industries, Ltd. and Tokyo Kasei Kogyo Co., Ltd., respectively.

BPT was obtained according to the following procedure. BPC $(1 \mathrm{~g}, 5 \mathrm{mmol})$ and $\mathrm{KSCN}(5 \mathrm{~g}$, $50 \mathrm{mmol}$ ) were dissolved in water $\left(50 \mathrm{~cm}^{3}\right)$, and the solution was continuously extracted with dichloromethane for five hours. The dichloromethane layer was separated, dried on anhydrous sodium sulfate and evaporated under reduced pressure to dryness. The residual matter was recrystalized from ethanol. The purity of the crystalline BPT thus obtained was determined to be $98.8 \%$ by analyzing the thiocyanate ion spectrophotometrically. ${ }^{5}$

\section{Electronic Spectra}

Electronic spectra were recorded on a Japan Spectroscopic Company Model UVIDEC-1 spectrophotometer and measured in a quartz cell having a $10 \mathrm{~mm}$ path length.

\section{Photopolymerization}

Solution photopolymerization was performed in a hard glass ampoule by the procedure described in a previous paper. ${ }^{1}$

Photopolymerization in the presence of a large quantity of water was performed by the following procedure. MMA, water, BPC, KSCN, and carbon tetrachloride were charged in a reaction vessel in Figure 1. The mixture was bubbled by blowing pure nitrogen through a glass filter from the bottom of the vessel and kept at $303 \pm 0.1 \mathrm{~K}$ in a water bath. After irradiation for prescribed period of time through a Pyrex glass cooling jacket using a Fuji Glass High Pressure Mercury Lamp HL-100

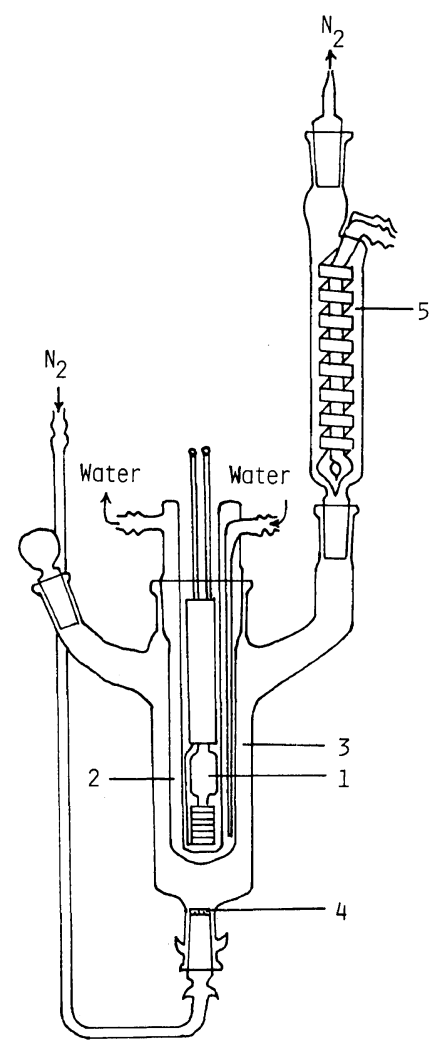

Figure 1. Photochemical reaction apparatus: 1, Fuji Glass High Pressure Mercury Lamp HL-100 (100 W); 2, cooling jacket; 3, reaction vessel; 4, glass filter; 5, Dimroth condenser.

$(100 \mathrm{~W})$, the reaction mixture was poured into a large quantity of methanol and the precipitated polymer was dried in vacuo.

\section{Number Average Molecular Weight}

The molecular weight of poly (methyl methacrylate) (PMMA) was determined in benzene at $310 \mathrm{~K}$ using a Knauel membran osmometer.

\section{RESULTS AND DISCUSSION}

\section{Photopolymerization of MMA with BPT in Dichloromethane}

The photopolymerization of MMA with BPT in dichloromethane was caried out under the same conditions as in the photopolymerization with BPB described in a previous paper. ${ }^{1}$ It was found that photopolymerization with BPT was similar to that 


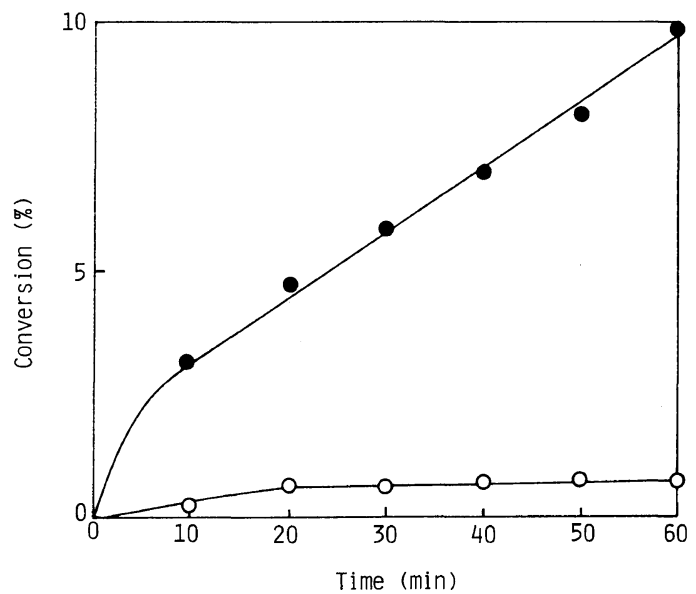

Figure 2. Photopolymerization of MMA with BPT in the presence of carbon tetrachloride: MMA, $5 \mathrm{~cm}^{3}$; BPT, $1 \times 10^{-3} \mathrm{moldm}^{-3} ; \mathrm{O}, \mathrm{CH}_{2} \mathrm{Cl}_{2}, 5 \mathrm{~cm}^{3} ; \mathrm{O}, \mathrm{CH}_{2} \mathrm{Cl}_{2}$, $4 \mathrm{~cm}^{3}$ and $\mathrm{CCl}_{4}, 1 \mathrm{~cm}^{3}$.

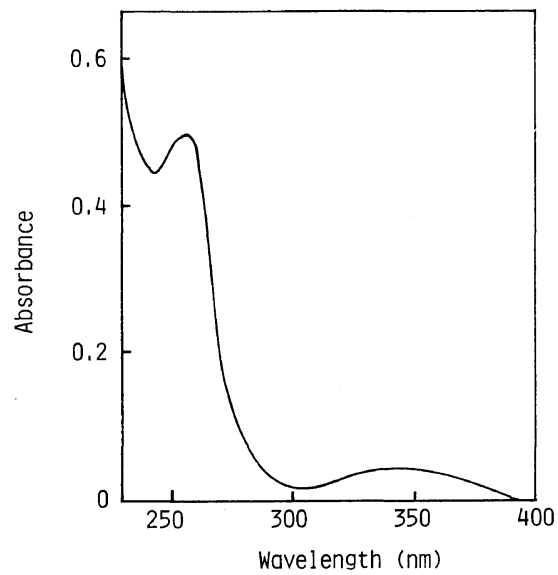

Figure 3. UV spectrum of BPT $\left(1 \times 10^{-4} \mathrm{~mol} \mathrm{dm}^{-3}\right)$ in dichloromethane.

with BPB with respect to the reaction mechanism. Photopolymerization with BPT in dichloromethane ceased below a $1 \%$ conversion (Figure 2) to give an oligo(methylmethacrylate) (OMMA), molecular weight determined by GPC to be 2,500. When MMA was irradiated with BPT in $\mathrm{CH}_{2} \mathrm{Cl}_{2}-\mathrm{CCl}_{4}$, however, the polymerization proceeded more rapidly than in $\mathrm{CH}_{2} \mathrm{Cl}_{2}$ (Figure 2) as was also the case with BPB. ${ }^{2}$ The UV spectrum of BPT in dichloromethane showed a CT band in a wavelength region from 320 to $380 \mathrm{~nm}$ (Figure 3), corresponding to that of BPB. ${ }^{1}$ Spectral changes by irradiation for

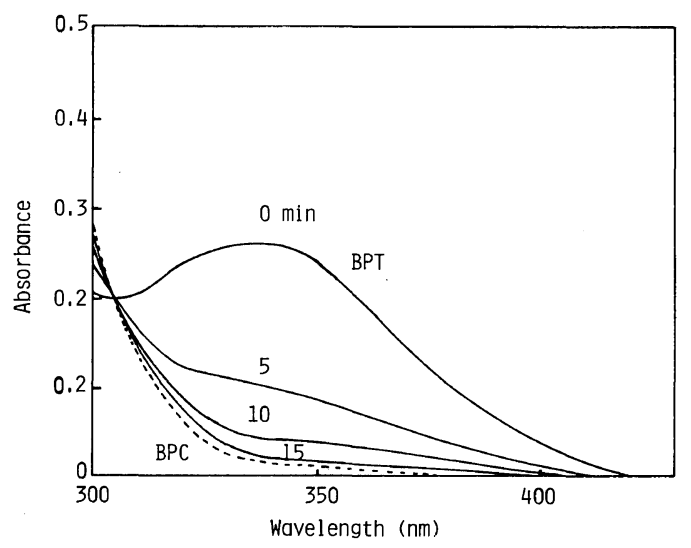

Figure 4. Electronic spectra of BPT during irradiation in the $\mathrm{MMA}-\mathrm{CH}_{2} \mathrm{Cl}_{2}-\mathrm{CCl}_{4}(5: 4: 1)$ mixture: BPT, $5 \times 10^{-4} \mathrm{~mol} \mathrm{dm}^{-3}$. Dotted line shows the spectrum of BPC $\left(5 \times 10^{-4} \mathrm{moldm}^{-3}\right)$ in $\mathrm{MMA}-\mathrm{CH}_{2} \mathrm{Cl}_{2}-\mathrm{CCl}_{4}$ $(5: 4: 1)$ without irradiation.

BPT in the MMA- $\mathrm{CH}_{2} \mathrm{Cl}_{2}-\mathrm{CCl}_{4}(5: 4: 1)$ mixture were also measured and the results, shown in Figure 4 , suggest that BPT is transformed into BPC by irradiation.

It was concluded that the photopolymerization of MMA with BPT in dichloromethane in the presence of carbon tetrachloride proceeds by the following methanism.
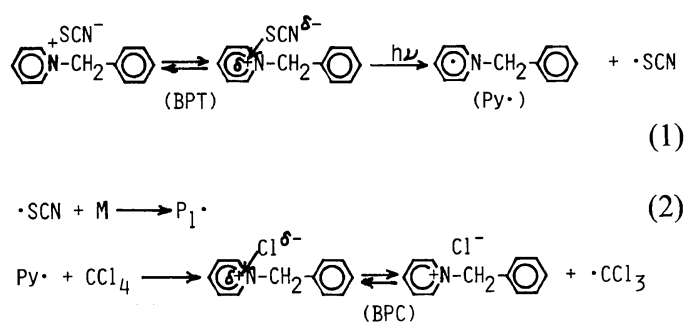

$\mathrm{BPC} \stackrel{\mathrm{h} \nu}{\longrightarrow} \mathrm{Py} \cdot+\cdot \mathrm{Cl}$

$\mathrm{Cl} \cdot\left(\right.$ or $\left.\cdot \mathrm{CCl}_{3}\right)+\mathrm{M} \longrightarrow \mathrm{P}_{1}$.

$\mathrm{P}_{\mathrm{n}} \cdot \mathrm{M} \longrightarrow \mathrm{P}_{\mathrm{n}+1}$.

$P_{n}+P_{m} \longrightarrow P_{n+m}$ or $P_{n}+P_{m}$

At the initial stage, MMA was initiated by $\cdot \mathrm{SCN}$ and $\cdot \mathrm{CCl}_{3}$ and then after about 20 minutes the initiating species were substantially replaced by $\mathrm{Cl} \cdot$ and $\cdot \mathrm{CCl}_{3}$ from the $\mathrm{BPC}-\mathrm{CCl}_{4}$ system. The details are described in another paper. ${ }^{2}$ 
Photopolymerization of $M M A$ with the $B P C-$ $\mathrm{KSCN}_{-} \mathrm{CCL}_{4}$ System in an Aqueous-Organic Two-Phase

MMA was mixed with an equal volume of water and the resulting mixture was separated into two phases, an upper organic layer and a lower aqueous layer. When BPC was added to the mixture, $92.6 \%$ of the fed BPC dissolved in the aqueous layer, as estimated by absorbance at $260 \mathrm{~nm}$. When a large excess of potassium thiocyanate (about twenty times the molar quantity of BPC) was added to the above mixture, $26.5 \%$ of the total $\mathrm{N}$ benzylpyridinium was spectrophotometrically found in the organic layer. These results indicate that BPT formed in the aqueous phase is appreciably transferred to the upper MMA phase. The mixture was placed in the photochemical reaction vessel shown in Figure 1 and irradiated for one hour. The results in Table I suggest that the polymerization of MMA was photoinitiated by BPT in the MMA phase. The molecular weight of the polymer obtained by the photopolymerization of MMA with the BPC-KSCN system in the aqueous-organic two-phase was larger than that obtained with BPT in dichloromethane. The reason for this may be that a small quantity of water in the MMA phase captured the pyridinyl radical, preventing it from terminating the polymerization, since pyridinyl radicals are known to react with water very quickly. ${ }^{6,7}$, The photopolymerization of MMA in the BPC-KSCN system in the aqueous-organic twophase was also accelerated by adding carbon tetrachloride, as shown in Table I. This indicates that

Table I. Photopolymerization of MMA in the $\mathrm{BPC}-\mathrm{KSCN}-\mathrm{CCl}_{4}$ system in an aqueousorganic two-phase

\begin{tabular}{|c|c|c|c|}
\hline BPC & $\mathrm{KSCN}$ & $\mathrm{CCl}_{4}$ & Conversion \\
\hline $\mathrm{g}(\mathrm{mmol})$ & $\mathrm{g}(\mathrm{mmol})$ & $\mathrm{cm}^{3}(\mathrm{mmol})$ & $\%$ \\
\hline - & - & - & 0.36 \\
\hline $0.02(0.097)$ & - & - & 0.24 \\
\hline - & $0.2(2.1)$ & - & 0.36 \\
\hline - & - & $2(21)$ & 0.84 \\
\hline $0.02(0.097)$ & - & $2(21)$ & 0.72 \\
\hline $0.02(0.097)$ & $0.2(2.1)$ & - & 1.40 \\
\hline $0.02(0.097)$ & $0.2(2.1)$ & $2(21)$ & 2.96 \\
\hline
\end{tabular}

MMA, $50 \mathrm{~cm}^{3} ; \mathrm{H}_{2} \mathrm{O}, 50 \mathrm{~cm}^{3}$; temp, $303 \mathrm{~K}$; time, $1 \mathrm{~h}$; blowing rate of $\mathrm{N}_{2}, 1 \mathrm{~cm}^{3} \mathrm{~s}^{-1}$. carbon tetrachloride captured the pyridinyl radical more rapidly than water, and that polymerization in the organic phase was presumably initiated by the BPT- $\mathrm{CCl}_{4}$ system as described in the preceding section. From these results, it is proposed that the photopolymerization of MMA with BPC-KSCN$\mathrm{CCl}_{4}$ system in aqueous-organic two-phase proceeds through the BPC-recycling mechanism expressed in Scheme 1.

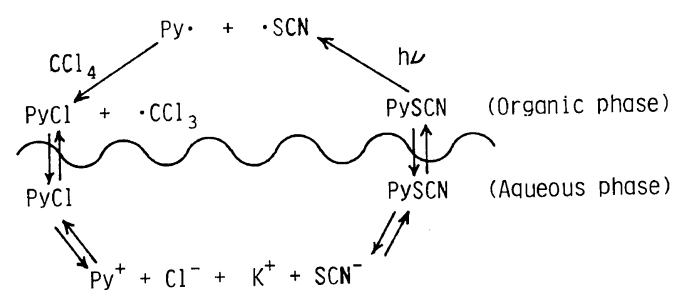

Scheme 1.

To confirm this mechanism, the photopolymerization of MMA in the BPC- $\mathrm{KSCN}-\mathrm{CCl}_{4}$ system was carried out at various feeding ratios of $\mathrm{BPC}$, $\mathrm{KSCN}$, and $\mathrm{CCl}_{4}$. Assuming a radical polymerization mechanism, $\left(R p^{2}-R p_{0}{ }^{2}\right)^{1 / 2}$ versus $(\mathrm{BPC})^{1 / 2}$ was plotted, where $R p$ is the apparent total rate of polymerization by the $\mathrm{BPC}-\mathrm{KSCN}-\mathrm{CCl}_{4}$ system, $R p_{0}$, that by $\mathrm{CCl}_{4}$ only, and (BPC), the quantity of fed BPC. The plot indicated a linear relation shown in Figure 5. A plot of $\left(R p^{2}-R p_{0}^{2}\right)^{1 / 2}$ versus $(\mathrm{KSCN})^{1 / 2}$ also indicated a linear relation shown in

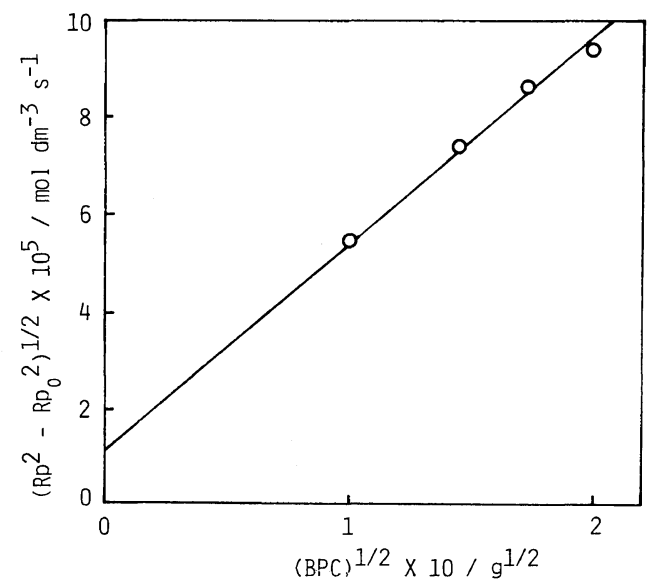

Figure 5. $\left(R p^{2}-R p_{0}{ }^{2}\right)^{1 / 2}$ versus $(\mathrm{BPC})^{1 / 2}$ for the photopolymerization of MMA in the $\mathrm{BPC}-\mathrm{KSCN}-\mathrm{CCl}_{4}$ system in an aqueous-organic two-phase. 


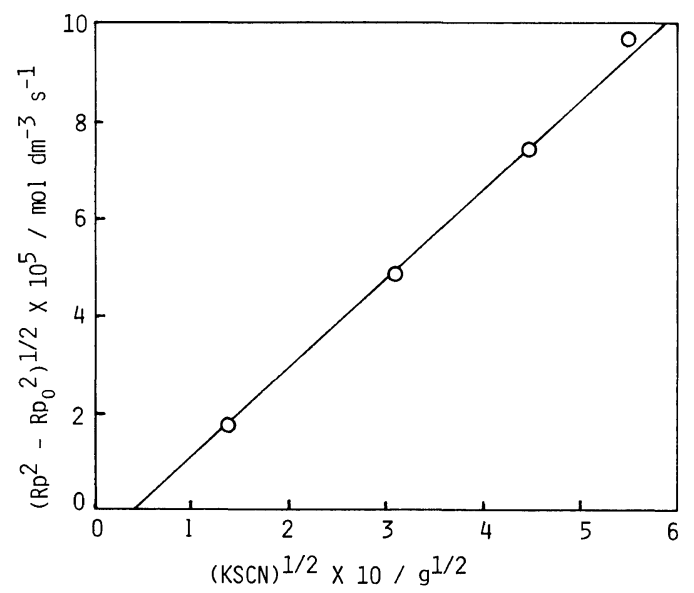

Figure 6. $\left(R p^{2}-R p_{0}{ }^{2}\right)^{1 / 2}$ versus $(\mathrm{KSCN})^{1 / 2}$ for the photopolymerization of MMA in the BPC-KSCN-CCl system in an aqueous-organic two-phase.

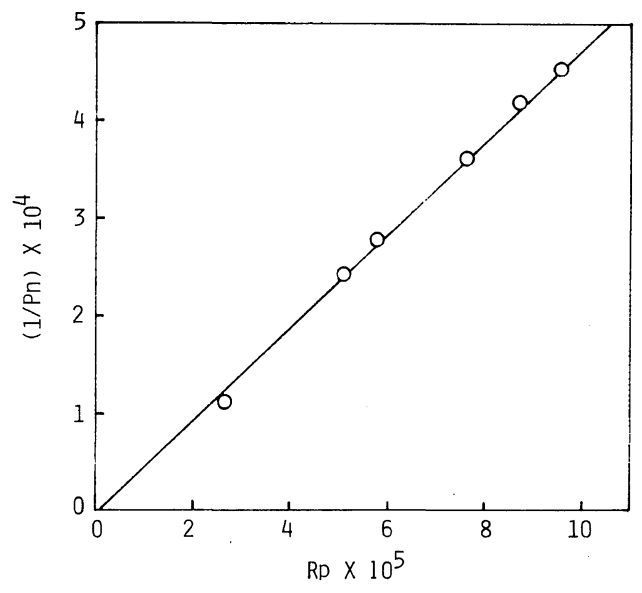

Figure 7. $1 / P n$ versus $R p$ for the photopolymerization of MMA in the BPC-KSCN-CCl ${ }_{4}$ system in an aqueous-organic two-phase.
Figure 6, where $(\mathrm{KSCN})$ is the quantity of fed $\mathrm{KSCN}$. The reciprocal of the degree of polymerization $(1 / P n)$ of PMMA thus obtained was plotted against $R p$. The plot indicated a linear relation, as shown in Figure 7. When the quantity of fed carbon tetrachloride was varied, conversion of the photopolymerization remained approximately constant, but the number average molecular weight slightly increases with the quantity of carbon tetrachloride as shown in Table II. This suggests that the reaction between $N$-benzylpyridinyl radical and carbon tetrachloride is too fast to have any influence on the rate of polymerization under the present experimental conditions and that carbon tetrachloride suppresses the termination of polymerization with the $N$-benzylpyridinyl radical and subsequently PMMA of high molecular weight was obtained.

From these results, it was concluded that the photopolymerization of MMA in the BPC- $\mathrm{KSCN}-$ $\mathrm{CCl}_{4}$ system was initiated in the organic phase by

Table II. Effects of $\mathrm{CCl}_{4}$ concentration on the photopolymerization of $\mathrm{MMA}$ in the $\mathrm{BPC}-\mathrm{KSCN}-\mathrm{CCl}_{4}$ system in an aqueous-organic two-phase

\begin{tabular}{lcc}
\hline \multicolumn{1}{c}{$\mathrm{CCl}_{4}$} & Conversion & \\
$\mathrm{cm}^{3}(\mathrm{mmol})$ & & $M_{n} \times 10^{-5}$ \\
\hline $0.01(0.10)$ & 2.49 & \\
$0.1(1.0)$ & 2.99 & 2.5 \\
$1(10)$ & 3.98 & 2.7 \\
$2(21)$ & 2.96 & 2.7 \\
$3(31)$ & 3.35 & 2.8 \\
$4(41)$ & 2.83 & 3.1 \\
\hline
\end{tabular}

MMA, $50 \mathrm{~cm}^{3} ; \mathrm{H}_{2} \mathrm{O}, 50 \mathrm{~cm}^{3} ; \mathrm{BPC}, 0.02 \mathrm{~g}(0.097$ mmol); KSCN, $0.2 \mathrm{~g}(2.1 \mathrm{mmol})$; temp, $303 \mathrm{~K}$; time, $1 \mathrm{~h}$; blowing rate of $\mathrm{N}_{2}, 1 \mathrm{~cm}^{3} \mathrm{~s}^{-1}$.

Table III. Photopolymerization of MMA in the BPC-KSCN-CCl $-\mathrm{Cl}_{4}-\mathrm{SDS}$ and $\mathrm{CPB}-\mathrm{KSCN}-\mathrm{CCl}_{4}$ systems in an aqueous-organic two-phase

\begin{tabular}{|c|c|c|c|c|}
\hline Surfactant & BPC & $\mathrm{KSCN}$ & $\mathrm{CCl}_{4}$ & Conversion \\
\hline $\mathrm{g}(\mathrm{mmol})$ & $\mathrm{g}(\mathrm{mmol})$ & $\mathrm{g}(\mathrm{mmol})$ & $\mathrm{cm}^{3}(\mathrm{mmol})$ & $\%$ \\
\hline SDS $0.014(0.047)$ & $0.02(0.097)$ & $0.2(2.1)$ & $2(21)$ & 15.40 \\
\hline SDS $0.014(0.047)$ & - & - & $2(21)$ & 1.36 \\
\hline CPB $0.02(0.057)$ & - & $0.2(2.1)$ & $2(21)$ & 21.76 \\
\hline CPB $0.02(0.057)$ & - & - & $2(21)$ & 1.89 \\
\hline
\end{tabular}

MMA, $50 \mathrm{~cm}^{3} ; \mathrm{H}_{2} \mathrm{O}, 50 \mathrm{~cm}^{3}$; temp, $303 \mathrm{~K}$; time, $1 \mathrm{~h}$; blowing rate of $\mathrm{N}_{2}, 1 \mathrm{~cm}^{3} \mathrm{~s}^{-1}$. 
$\cdot \mathrm{SCN}$ and $\cdot \mathrm{CCl}_{3}$ produced from the reactions shown in Scheme 1.

If the interface area between the aqueous and organic phases becomes larger, BPT formed in the aqueous phase might be transferred more quickly to the organic phase. To confirm this, the polymerization of MMA by the BPC- $\mathrm{KSCN}-\mathrm{CCl}_{4}$ system in the aqueous-organic two-phase was carried out in the presence of SDS as a surfactant. In this emulsified system, conversion following irradiation for one hour was as large as anticipated, but without BPC-KSCN, no polymerization occurred (Table III). The rate of polymerization undoubtedly increased with an increase in SDS concentration, but at a higher concentration of SDS, the polymerization system became very foamy by blowing nitrogen gas through it. Therefore, SDS was added only at a concentration of $0.047 \mathrm{mmol}$ per $50 \mathrm{~cm}^{3}$ of water. Other surfactants, such as sodium dodecylbenzenesulfonate, sodium laurate and cetyltrimethylammonium bromide were also effective. As long as the concentration of surfactant among the emulsion particles was not too large, acceleration of the polymerization by the surfactant depended only on the area of the interface between the aqueous and organic phases and was independent of the type of surfactant. When CPB was used as the surfactant without BPC, the photopolymerization of MMA proceeded as quickly as that in the SDS-BPC$\mathrm{KSCN}-\mathrm{CCl}_{4}$ system. The photopolymerization of MMA with CPB is now being investigated at our laboratory.

\section{REFERENCES}

1. K. Tabuchi and N. Sakota, Polym. J., 15, 57 (1983).

2. K. Tabuchi and N. Sakota, Polym. J., 15, 569 (1983).

3. J. F. Coetzee and J. J. Campion, J. Am. Chem. Soc., 89, 2517 (1967).

4. K. Nishihara and N. Sakota, Makromol. Chem., 165, 105 (1973).

5. Nippon Bunseki Kagaku Kai, "Bunseki Kagaku Binran," Maruzen Co., Tokyo, 1971, p 174.

6. E. M. Kosower and A. Teuerstein, J. Am. Chem. Soc., 95, 6127 (1973).

7. E. M. Kosower and E. J. Poziomek, J. Am. Chem. Soc., 86, 5515 (1964). 